\title{
Exposure Response Supports Therapeutic Drug Monitoring for Dabigatran Etexilate in Patients with Atrial Fibrillation
}

\author{
Bryan H. Simpson ${ }^{1}$ David M. Reith ${ }^{2}$ Natalie J. Medlicott ${ }^{1} \quad$ Alesha J. Smith $^{1}$ \\ ${ }^{1}$ School of Pharmacy, University of Otago, Dunedin, New Zealand \\ 2 Dean's Department, Dunedin Medical School, University of Otago, \\ Dunedin, New Zealand \\ Address for correspondence Bryan H. Simpson, BSc, BPharm, \\ PGDipPharm, School of Pharmacy, Adams Building, 18 Frederick \\ Street, PO Box 56, Dunedin, 9054, New Zealand \\ (e-mail: bryan.simpson@otago.ac.nz).
}

TH Open 2019;3:e210-e215.

Abstract

Keywords
- dabigatran etexilate
- therapeutic drug
monitoring
- hemorrhage
- stroke
- population
pharmacokinetic

Background Dabigatran etexilate has become widely used for the prevention of stroke in patients with nonvalvular atrial fibrillation (NVAF). Currently, there is limited information in real-world patients relating to dabigatran etexilate exposure and response.

Methods This retrospective cohort study used administrative health data for NVAF patients dispensed dabigatran etexilate between July 1, 2011 and December 31, 2015. Outcomes of cerebrovascular accident (CVA), systemic embolism, and hemorrhage were extracted. Simulated pharmacokinetic parameters were obtained using a published population pharmacokinetic model of dabigatran etexilate. Area under the curve calculated for a 24-hour period at steady state $\left(A \cup C_{s s}\right.$ ), the exposure parameter, was derived using these simulations and the dosing data and the exposure-response relationship were investigated. The risk of adverse outcomes at $A \cup C_{s s}$ quartiles was compared using Poisson regression and expressed using incidence rate ratios ( $95 \%$ confidence interval) adjusted for known potential confounders.

Results In total, 2,660 NVAF patients had been dispensed dabigatran etexilate. For these patients there was a decreased risk of hemorrhage $(0.51,0.32-0.79)$ when dabigatran $A \cup C_{s s}$ was in the second quartile range of 1.70 to $1.96 \mathrm{mg} \mathrm{h} / \mathrm{L}$ and thromboembolism/CVA $(0.34,0.16-0.76)$ when in the third quartile range of 1.97 to $2.26 \mathrm{mg} \mathrm{h} / \mathrm{L}$. An increased risk of hemorrhage $(1.68,1.18-2.38)$ was observed when $\mathrm{AUC}_{\mathrm{ss}}$ was in the fourth quartile range of 2.27 to $12.76 \mathrm{mg} \mathrm{h} / \mathrm{L}$.

Conclusion An exposure-response relationship for dabigatran etexilate was described, where the most effective response was observed when $A U C_{s s}$ was in the range of 1.70 to $2.26 \mathrm{mg} \mathrm{h} / \mathrm{L}$. Hence, it is feasible to develop guidance for optimal dosing to improve outcomes for patients with NVAF.

\section{Background}

The direct oral anticoagulant (DOAC) dabigatran etexilate has become widely used since its approval for the prevention of stroke in patients with nonvalvular atrial fibrillation (NVAF), ${ }_{1}$ but has been associated with several adverse outcomes. ${ }^{2,3}$

received

January 24, 2019 accepted after revision June 7, 2019
DOI https://doi.org/

10.1055/s-0039-1693486. ISSN 2512-9465.
Despite this there is paucity of information for the dose response for this important medication.

Dabigatran etexilate is normally administered as a twicedaily fixed-dose regimen with the dosage modified by age and/or creatinine clearance, use of concomitant drugs, and thromboembolic risk versus bleeding risk. ${ }^{4}$ As with other

(c) 2019 Georg Thieme Verlag KG Stuttgart · New York
License terms

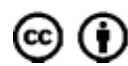


DOAC medications, dabigatran etexilate exhibits a more predictable pharmacokinetic and pharmacodynamics profile when compared with vitamin $\mathrm{K}$ antagonists. ${ }^{5}$

Although dabigatran etexilate has been promoted as not requiring routine coagulation monitoring, this has become controversial. ${ }^{6}$ Certainly, there are specific clinical situations where assessment of the anticoagulation effect may be required, such as: for those who are bleeding, before and after administration of the dabigatran-specific antidote idarucizumab (Praxbind), evaluation of therapy failure in case of thrombosis, renal failure, before emergency surgery, before potential thrombolysis in ischemic stroke, at extremes of bodyweight, concomitant use of drugs known to affect pharmacokinetics of dabigatran etexilate, and in cases of suspected nonadherence. ${ }^{7}$ Moreover, it has been reported that if therapeutic drug monitoring (TDM) was undertaken major bleeds could be reduced by 30 to $40 \%$ when compared with well-controlled warfarin. ${ }^{6}$

Currently, the Sponsor indicates that an increased risk of bleeding can possibly be detected via elevated coagulation tests such as thrombin time (TT), ecarin clotting time (ECT), and activated partial thromboplastin time (aPTT). ${ }^{8}$ However, there are limitations to the aPTT, such as the test having limited sensitivity making it unsuitable for precise quantification of the anticoagulant effect and the ECT test not being readily available or useful in the absence of standardization means that there is limited utility of these tests in clinical practice. ${ }^{9}$ TT is a very useful test for detecting low levels of dabigatran etexilate in plasma. ${ }^{10}$ However, TT becomes rapidly unclottable in the presence of low dabigatran etexilate concentrations, and therefore cannot be used for the overall expected drug concentration measurement. ${ }^{11}$ In relation to treatment failure, it has been reported that the Sponsor believes that due to the low number of endpoint events for venous thromboembolism (VTE) patients and the availability of only pharmacokinetic data from clinical trials, only a limited exposure-response analysis could be undertaken for VTE prevention. ${ }^{12}$ Therefore, there has been no guidance provided for monitoring patients for possible subtherapeutic treatment. ${ }^{12}$

Despite it now being feasible to determine dabigatran plasma concentrations, ${ }^{13,14}$ the thresholds are yet to be validated to ensure that clinical decisions based on the plasma concentrations represent the balance between avoiding bleeding and preventing thrombosis. ${ }^{9}$ While it has been shown that there is an association between plasma concentrations and bleeding risk, the clear cut-offs for bleeding and thromboembolism/cerebrovascular accident (CVA) risk are not yet established. ${ }^{9}$ With no reported reference ranges for dabigatran etexilate TDM, further studies would help clarify this issue.

The aim of the present study was to investigate the relationship between dabigatran etexilate exposure and adverse response in real-world patients.

\section{Methods}

\section{Identification of Study Cohort}

This was a retrospective cohort study using administrative health data from New Zealand. The databases accessed were the Best Practice Intelligence (BPI) database operated by Best Practice Advocacy Centre Clinical Solutions, New Zealand ${ }^{15}$ and the New Zealand Ministry of Health Pharmaceutical Collection ${ }^{16}$ (PC). The BPI database is a secure, internet-based, reporting tool that uses data downloaded from the enrolled general practice patient electronic health record (EHR) and covers approximately $20 \%$ of the New Zealand population. The PC contains prescription details about pharmaceutical dispensing claims for dabigatran etexilate along with other prescribed medicines as well as information on gender, date of birth, age, ethnicity, deprivation index score, frequency, and quantity dispensed for all of the New Zealand population. The study population included patients with a diagnosis of NVAF, aged 18 years or older, had at least one dispensing of dabigatran etexilate during the study period between July 1, 2011 (when dabigatran etexilate became available in New Zealand) and December 31, 2015, serum creatinine measurements within 60 days before or 30 days after their first dispensing of dabigatran etexilate, and bodyweight measurements within 1 year before or after their first dispensing of dabigatran etexilate. If a patient had a diagnosis of VTE or deep-vein thrombosis concurrently recorded with NVAF during the study period, they were excluded. The information from different datasets were linked using each patient's encrypted National Health Index number (NHI; a life-long unique identifier for all interactions with the New Zealand health system) to ensure patient anonymity. Ethical approval was obtained from the University of Otago, New Zealand Ethics Committee (reference: HD15/054).

\section{Patient Covariates}

Dispensed medications, patient demographic, and covariate data were extracted from the PC and BPI databases for those who meet the inclusion criteria. Data related to medications that are reported to have an impact on area under the curve (AUC; verapamil, amiodarone, and proton pump inhibitors) ${ }^{17}$ when taken concomitantly with dabigatran etexilate were extracted from the PC database for patients who had both medications dispensed within 90 days. As it has also been reported that patients with heart failure have an increase in AUC, ${ }^{17}$ data related to whether a patient has a diagnosis of heart failure while being treated with dabigatran etexilate were extracted from the BPI database. Patients were stratified into age groupings of under 65 years, 65 to 74 years, 75 to 79 years, and over 80 years to align to both regulatory and the categories used by the Sponsor to guide dosing. ${ }^{4,18,19}$

\section{Estimation of Renal Function}

If multiple serum creatinine or bodyweight measurements were recorded, the measurement closest to the initiation of dabigatran etexilate initiation was used. Weight measurements more than 5 standard deviations from the mean were considered to be due to data-entry errors and were excluded.

Baseline renal function was estimated via the CockcroftGault equation using Eq. 1: 


$$
\mathrm{CrCl}(\mathrm{mL} / \mathrm{min})=\frac{(140-\text { Age }) \times \text { Weight }}{72 \times \mathrm{SCr}} \times 0.85[\text { if female }]
$$

where $\mathrm{CrCl}=$ creatinine clearance; age = age in years; weight $=$ weight in $\mathrm{kg}$; $\mathrm{SCr}=$ serum creatinine (expressed in $\mathrm{mg} / \mathrm{dL}$ ).

Eq. 1

Derived area under the curve at steady state ( $A U C_{s s}$ ) Simulated pharmacokinetic parameters (volume of distribution, bioavailability, and clearance) were obtained using a previously published population pharmacokinetic two-compartment model of dabigatran etexilate. ${ }^{17}$ Simulated individual pharmacokinetic parameters for the first compartment [clearance $(\mathrm{CL})$, central volume of distribution (V1) and bioavailability (F)] were determined for patients included in the study cohort using the previously published nonlinear mixedeffects model using Phoenix NLME Version 7. AUC $_{\mathrm{ss}}$ for a 24hour period, the exposure parameter, was derived using these simulated estimates and the dosing data obtained from the PC. The second-compartment parameters were fixed (intercompartmental clearance $[Q=35.5 \mathrm{~L} / \mathrm{h}]$ and volume distribution of peripheral compartment $\left[V_{\text {per }}=345 \mathrm{~L}\right]$ ).

\section{Patient Outcomes}

The outcomes of interest were ${ }^{1}$ any admission to hospital for hemorrhage or $^{2}$ thromboembolism/CVA. These were extracted from the New Zealand Ministry of Health National Minimum Dataset (NMDS). ${ }^{20}$ The NMDS is the national record of all public and private hospital discharge information, including coded clinical data for admissions longer than 4 hours for all of the New Zealand population. This database uses the International Statistical Classification of Diseases and Related Health Problems Tenth Revision, Australian Modification (ICD-10-AM) to record the diagnosis. ${ }^{21}$ The date of admission to hospital was also extracted and aligned with the preceding dispensing of dabigatran etexilate for that patient. Patients were followed from their first dispensing of dabigatran etexilate until the date of hospitalization, cessation of dabigatran etexilate treatment, or study end.

\section{Statistical Analyses}

Continuous variables were tested for normal distribution by the skewness and kurtosis test. Normally distributed data are presented as the mean \pm standard deviation and nonnormally distributed data as the median (interquartile range, IQR). Categorical variables were expressed as percentages.

To evaluate the exposure-response relationship, patients were stratified into $\mathrm{AUC}_{\mathrm{sS}}$ exposure quartiles (Q1-Q4). As there were differences in individual patient follow-up time, the risk of hemorrhage or thromboembolism/CVA between $\mathrm{AUC}_{\mathrm{ss}}$ quartiles was compared using Poisson regression. ${ }^{22}$ The resulting estimates were expressed using incidence rate ratios (IRRs; $95 \%$ confidence intervals [CIs]). ${ }^{22}$ As exposure changed over time the standard hazard functions could not be fitted and rates were calculated per unit time exposure from which rate ratios (which are unitless) were calculated. Models were adjusted for known potential pharmacodynamic confounders: gender, age
75 to 79 years, age 80 years and over, Māori and Pacific peoples ethnicities, and deprivation rating (continuous). ${ }^{23}$ Results were considered statistically significant if $p<0.05$.

Statistical analyses were performed using Stata/IC (Version 14.2, StataCorpLP, TX, United States).

\section{Results}

\section{Patient Characteristics}

There were 2,660 individual patients identified in the databases who had been dispensed dabigatran etexilate with a diagnosis of atrial fibrillation (AF), aged 18 years or more, and who had one or more serum creatinine and bodyweight measurement recorded. The median age of patients in this cohort was 73 years (IQR: 66-79.5 years) and 1,525 (57.3\%) were male. The median bodyweight was $85 \mathrm{~kg}$ (IQR: 72-100 kg). The median creatine clearance was $74.1 \mathrm{~mL} / \mathrm{min}$ (IQR: 56.1-98.2). Overall patient covariate information is displayed in -Table 1.

\section{Patient Outcomes}

Approximately $4 \%$ of patients required hospitalization due to an adverse event occurring while being treated with dabigatran etexilate; there were 87 (3.3\%) hemorrhagic events with a median age of 74 years (IQR: $70-81$ years) and 29 (1.1\%) thromboembolic/CVA events with a median age of 72 years (IQR: 68-79 years).

\section{Exposure-Derived Area Under the Curve}

There were 65,233 individual prescriptions for dabigatran etexilate with a total 5,149 person-years supplied. The median $\mathrm{AUC}_{\mathrm{ss}}$ was $2.0 \mathrm{mg}$ h/L (IQR: $1.7-2.3 \mathrm{mg} \mathrm{h} / \mathrm{L}$ ) and when stratified by quartile each had the following results:

- Q1 had a mean of $1.48 \mathrm{mg} \mathrm{h} / \mathrm{L}$ (range: 0.79-1.69 mg h/L),

- Q2 had a mean of $1.83 \mathrm{mg} \mathrm{h} / \mathrm{L}$ (range: $1.70-1.96 \mathrm{mg} \mathrm{h} / \mathrm{L}$ ),

- Q3 had a mean of $2.11 \mathrm{mg} \mathrm{h} / \mathrm{L}$ (range: $1.97-2.26 \mathrm{mg} \mathrm{h} / \mathrm{L}$ ),

- Q4 had a mean of $2.74 \mathrm{mg} \mathrm{h} / \mathrm{L}$ (range: $2.27-12.76 \mathrm{mg} \mathrm{h} / \mathrm{L}$ ).

Simulated pharmacokinetic parameters are summarized in - Table 2. Those with an $\mathrm{AUC}_{\mathrm{ss}}$ in the second and third quartiles had a reduced risk of hemorrhage [IRR: $0.51 ; 95 \% \mathrm{CI}$ : $0.32-0.79 ; p=0.003$ ] and thromboembolism/CVA [IRR: 0.34; 95\% CI: $0.16-0.76 ; p=0.008$ ] respectively, while those with an $\mathrm{AUC}_{\mathrm{ss}}$ in the fourth quartile had an increased risk of hemorrhage [IRR: 1.68 ; 95\% CI: 1.18-2.38; $p=0.004$ ] (-Table 3).

\section{Discussion}

This study demonstrates the feasibility of using measures of dabigatran etexilate pharmacokinetic exposure to optimize dosing and potentially improve patient outcomes.

The present study utilizes a novel approach of combining individual patient data, treatment outcomes, and a previously developed population pharmacokinetic model of dabigatran etexilate. $\mathrm{AUC}_{\mathrm{ss}}$ data for each individual patient were derived and adjusted IRR at each $\mathrm{AUC}_{\mathrm{ss}}$ quartile calculated. We observed that there was a greater risk for a hemorrhage observed for those patients with an $A_{U S C}$ in the fourth quartile, while there was a protective effect observed in 
Table 1 Individual patient covariate information

\begin{tabular}{|c|c|c|c|}
\hline Covariate & $n$ & $\%$ & Total exposure (person-years) \\
\hline \multicolumn{4}{|l|}{ Sex } \\
\hline Male & 1,525 & 57.3 & 3,023 \\
\hline Female & 1,135 & 42.7 & 2,156 \\
\hline \multicolumn{4}{|l|}{ Age } \\
\hline$<65 y$ & 554 & 20.8 & 952 \\
\hline $65-74 y$ & 922 & 34.7 & 1,836 \\
\hline $75-79$ y & 519 & 19.5 & 1,043 \\
\hline$>80 y$ & 665 & 25.0 & 1,318 \\
\hline \multicolumn{4}{|l|}{ Ethnicity } \\
\hline European & 2,098 & 78.9 & 4,075 \\
\hline Māori & 315 & 11.8 & 587 \\
\hline Pacific peoples & 65 & 2.4 & 129 \\
\hline Asian & 24 & 0.9 & 63 \\
\hline MELAA & 3 & 0.1 & 7 \\
\hline Other ethnicity & 155 & 5.8 & 287 \\
\hline Heart failure & 411 & 15.5 & 845 \\
\hline \multicolumn{4}{|l|}{ Renal status } \\
\hline Severe impairment $(\mathrm{CrCl}<30 \mathrm{~mL} / \mathrm{min})$ & 43 & 1.6 & 58 \\
\hline Moderate impairment $(30 \leq \mathrm{CrCl}<50 \mathrm{~mL} / \mathrm{min})$ & 425 & 16.0 & 732 \\
\hline Mild impairment $(50 \leq \mathrm{CrCl}<80 \mathrm{~mL} / \mathrm{min})$ & 1,036 & 39.0 & 2,081 \\
\hline No impairment $(80 \leq \mathrm{CrCl}<120 \mathrm{~mL} / \mathrm{min})$ & 818 & 30.8 & 1,631 \\
\hline No impairment $(\mathrm{CrCl}>120 \mathrm{~mL} / \mathrm{min})$ & 338 & 12.7 & 647 \\
\hline \multicolumn{4}{|l|}{ Co-prescribed medications } \\
\hline Verapamil & 42 & 1.6 & 85 \\
\hline Amiodarone & 165 & 6.2 & 257 \\
\hline Proton pump inhibitor & 1,186 & 44.6 & 2,268 \\
\hline \multicolumn{4}{|l|}{ New Zealand deprivation score } \\
\hline 1-Most deprived & 154 & 5.8 & 299 \\
\hline 2 & 165 & 6.2 & 311 \\
\hline 3 & 224 & 8.4 & 471 \\
\hline 4 & 206 & 7.7 & 372 \\
\hline 5 & 183 & 6.9 & 346 \\
\hline 6 & 212 & 8.0 & 397 \\
\hline 7 & 224 & 8.4 & 430 \\
\hline 8 & 192 & 7.2 & 372 \\
\hline 9 & 232 & 8.7 & 458 \\
\hline 10-Least deprived & 200 & 7.5 & 378 \\
\hline Not recorded & 668 & 25.1 & 1,315 \\
\hline \multicolumn{4}{|l|}{ Formulation dispensed (at baseline) } \\
\hline $75 \mathrm{mg}$ & 43 & 1.6 & 2 \\
\hline $110 \mathrm{mg}$ & 1,375 & 51.7 & 127 \\
\hline $150 \mathrm{mg}$ & 1,242 & 46.7 & 95 \\
\hline \multicolumn{4}{|l|}{ Formulation dispensed (for all dispensings) } \\
\hline $75 \mathrm{mg}$ & 1,189 & 1.8 & 80 \\
\hline $110 \mathrm{mg}$ & 34,670 & 53.1 & 2,631 \\
\hline $150 \mathrm{mg}$ & 29,374 & 45.0 & 2,438 \\
\hline
\end{tabular}

Abbreviation: MELAA, Middle Eastern, Latin American or African ethnicity. 
e214 Exposure Response Supports TDM for Dabigatran Etexilate in AF Patients Simpson et al.

Table 2 Summary of simulated pharmacokinetic parameters $(n=65,233)$

\begin{tabular}{|l|l|l|l|}
\hline $\begin{array}{l}\text { Simulated } \\
\text { parameter }\end{array}$ & Mean (SD) & Median & IQR \\
\hline $\mathrm{CL}(\mathrm{ml} / \mathrm{min})$ & $69.3(17.6)$ & 68.8 & $56.9-81.2$ \\
\hline$V_{1}(\mathrm{~L})$ & $710.1(117.6)$ & 697.4 & $630.0-775.1$ \\
\hline $\mathrm{F}$ & $1.0(0.1)$ & 1.0 & $0.9-1.0$ \\
\hline
\end{tabular}

Abbreviations: CL, clearance; F, bioavailability; IQR, interquartile range; $\mathrm{SD}$, standard deviation; $V_{1}$, central volume of distribution.

Note: Fixed parameters; $Q(35.5 \mathrm{~L} / \mathrm{h})$ and $V_{\text {per }}(345 \mathrm{~L})$.

the second quartile. In relation to thromboembolism, there was also a protective effect observed for those patients with an $\mathrm{AUC}_{\mathrm{ss}}$ in the third quartile. Therefore, adjusting doses to achieve an $\mathrm{AUC}_{\mathrm{ss}}$ that is in the range of 1.70 to $2.26 \mathrm{mg} \mathrm{h} / \mathrm{L}$ may result in better outcomes for patients.

Additionally, the median age of our cohort was 73 years, which is older than that of patients enrolled in the dabigatran etexilate clinical trial. ${ }^{24}$ It is acknowledged in the literature that elderly patients are at an overall increased risk of adverse outcomes with other studies having demonstrated that increasing age was a risk factor for having increased levels of dabigatran etexilate. ${ }^{13,25}$ Therefore, the present study is able to provide a basis for developing reference ranges for optimal dosing for this at-risk and understudied population.

Currently it is not clear if TDM would be cost-effective in the clinical setting. It has been recently reported that with the rapidly growing use of DOACs there is increasing debate about the utility of point-of-care testing. ${ }^{13}$ Despite these tests being available they are more expensive than traditional coagulation tests which may limit their utilization. With traditional coagulation tests having limited use in clinical practice, due to either poor sensitivity or standardization, ${ }^{9}$ it has been suggested that point-of-care use in selected clinical situations may counterbalance their cost. ${ }^{13}$ Additionally, to achieve the target $\mathrm{AUC}_{\mathrm{ss}}$, the dosing schedule may need altering (for example, extending or reducing dose intervals or combining different available formulation strengths) and dose simulations might clarify this point. Therefore, the present study can aid in the future economic evaluation for cost-effectiveness of TDM utility for dabigatran etexilate by providing treatment reference ranges conducive to favorable patient outcomes.
This study provides a preliminary clinical therapeutic reference range for dabigatran etexilate to help guide further studies investigating optimal dosing. Additionally, we are able to provide a real-world study population that has sufficient numbers to examine subtherapeutic treatment, a gap in knowledge identified in the literature. ${ }^{12}$ With dabigatran etexilate TDM suggested to possibly reduce major bleeds by 30 to $40 \%$, compared with well-controlled warfarin, ${ }^{6}$ this study expands on this by providing a reference range that could be translated into the clinical setting along with a possible decrease in thrombotic events.

The limitations of this study include the NMDS only capturing patient data for those who require in-patient hospitalization for a duration of more than 4 hours. Therefore, any outcomes of interest that did not meet these criteria, for example, a hemorrhage or thromboembolism/CVA that resulted in death without an in-patient hospitalization, would not be included in the dataset resulting in possible underestimations. Additionally, there is the possibility of errors in the clinical information from the NMDS and primary-care dataset. These errors could result in inclusion or exclusion of clinical outcomes of interest. Although, it has been reported that there is high sensitivity when using the International Classification of Diseases, Ninth Revision, Clinical Modification (ICD-9-CM) to identify hemorrhagic events with $93 \%$ sensitivity and $88 \%$ specificity to identifying a definite major hemorrhagic event. ${ }^{26}$ Similarly, it has been reported that using ICD-10 to identify thromboembolism/CVA has a positive predictive value (PPV) of close to or greater than $90 \%$ and therefore adequate to identify thromboembolism/CVA. ${ }^{27}$ We assumed that the ICD-10-AM used to identify hemorrhagic events had high sensitivity and specificity and those used to identify thromboembolism/CVA had a high PPV. These limitations contribute to background variability but would not be expected to contribute to a systematic bias. Additionally, the PC only provides information related to the dispensing of medications and it is not possible to confirm if the patients within this cohort have adhered to the prescribed regimen. Also, the population pharmacokinetic model utilized did not include covariate effects for other drug interactions (such as atorvastatin, rifampicin, clarithromycin) as there were no published validated models. Although risk factors were included in the exposure model utilized, HAS-BLED and CHA2DS2-VASc covariates were not. However, known risk factors in our population were included (such as ethnicity,

Table 3 Poisson regression expressed as adjusted incidence rate ratios (IIRs) and 95\% confidence interval (95\% CI) of hemorrhage and thromboembolism/CVA by UUC $_{\text {ss }}$ quartiles $(n=65,233)$

\begin{tabular}{|l|l|l|l|l|l|l|}
\hline & \multicolumn{4}{|l|}{ Hemorrhage } & \multicolumn{4}{l|}{ Thromboembolism/CVA } \\
\hline AUC $_{\text {ss }}$ & IRR $(95 \% \mathrm{Cl})$ & $\boldsymbol{z}$ & $\boldsymbol{p}>|\mathbf{z}|$ & IRR $(95 \% \mathrm{CI})$ & $\boldsymbol{z}$ & $\boldsymbol{p}>|\mathbf{z}|$ \\
\hline Quartile 1 & $0.9(0.61-1.33)$ & -0.52 & 0.606 & $1.31(0.73-2.34)$ & 0.89 & 0.371 \\
\hline Quartile 2 & $0.51^{\mathrm{a}}(0.32-0.79)$ & -3.01 & 0.003 & $1.49(0.87-2.55)$ & 1.45 & 0.146 \\
\hline Quartile 3 & $1.13(0.81-1.59)$ & 0.71 & 0.478 & $0.34^{\mathrm{a}}(0.16-0.76)$ & -2.66 & 0.008 \\
\hline Quartile 4 & $1.68^{\mathrm{a}}(1.18-2.38)$ & 2.89 & 0.004 & $1.20(0.68-2.12)$ & 0.62 & 0.536 \\
\hline
\end{tabular}

Abbreviations: $A U C_{s s}$, area under the curve at steady state; CVA, cerebrovascular accident; IRR, adjusted incidence rate ratio for potential confounders: gender, age 75 years and over, Māori and Pacific peoples ethnicities and deprivation rating (continuous).

${ }^{\text {a }}$ Statistically significant. 
increasing age, and deprivation score). The main strength of this study is the inclusion of a large cohort of patients with sufficient sample size to provide adequate information about dabigatran etexilate exposure response.

\section{Conclusion}

This retrospective cohort demonstrated that there is a relationship between dabigatran etexilate exposure and adverse response in real-world patients. It has established that it is feasible to provide guidance for optimal dosing to improve outcomes for patients with AF. This is particularly relevant for elderly patients who are at greater risk of adverse treatment outcomes.

\section{Conflict of Interest \\ None declared.}

\section{References}

1 Simpson B, Reith D, Medlicott N, et al. Utilisation of New Zealand primary care electronic data to guide clinical decision support system design-a retrospective cohort for dabigatran. Clin Ther 2017;39:e7

2 Bell S, Nand J, Spriggs D, Young L, Dawes M. Initial experience with dabigatran etexilate at Auckland City Hospital. N Z Med J 2012; 125(1349):105-107

3 Thorne K, Jayathissa S, Dee S, et al. Adherence and outcomes of patients prescribed dabigatran (Pradaxa) in routine clinical practice. Intern Med J 2014;44(03):261-265

4 Boehringer Ingelheim (N.Z.) Limited. Pradaxa: New Zealand Datasheet. Available at: http://www.medsafe.govt.nz/other/copyright. asp. Accessed November 2017

5 Dale BJ, Chan NC, Eikelboom JW. Laboratory measurement of the direct oral anticoagulants. Br J Haematol 2016;172(03):315-336

6 Cohen D. Dabigatran: how the drug company withheld important analyses. BMJ 2014;349:g4670

7 Comuth WJ, Henriksen LØ, van de Kerkhof D, et al. Comprehensive characteristics of the anticoagulant activity of dabigatran in relation to its plasma concentration. Thromb Res 2018;164:32-39

8 Boehringer Ingelheim International $\mathrm{GmbH}$. Pradaxa $110 \mathrm{mg}$ hard capsules. Available at: https://www.medicines.org.uk/emc/product/6229/smpc\#companyDetails. Accessed September 5, 2018

9 Douxfils J, Ageno W, Samama CM, et al. Laboratory testing in patients treated with direct oral anticoagulants: a practical guide for clinicians. J Thromb Haemost 2018;16(02):209-219

10 Chin PKL, Wright DFB, Patterson DM, Doogue MP, Begg EJ. A proposal for dose-adjustment of dabigatran etexilate in atrial fibrillation guided by thrombin time. Br J Clin Pharmacol 2014;78 (03):599-609

11 Amiral J, Dunois C, Amiral C, Seghatchian J. An update on laboratory measurements of Dabigatran: smart specific and calibrated dedicated assays for measuring anti-Ila activity in plasma. Transfus Apheresis Sci 2016;54(03):428-437
12 Committee for Medicinal Products for Human Use (CHMP). Assessment report. Pradaxa. International non-proprietary name: dabigatran etexilate. 2014. Available at: https://www.ema.europa.eu/ en/documents/variation-report/pradaxa-h-c-829-ii-0048-g-eparassessment-report-variation_en.pdf. Accessed June 27, 2019

13 Rottenstreich A, Zacks N, Kleinstern G, et al. Direct-acting oral anticoagulant drug level monitoring in clinical patient management. J Thromb Thrombolysis 2018;45(04):543-549

14 Poli S, Härtig F, Spencer C, et al. Diagnostic accuracy of a novel chromogenic direct thrombin inhibitor assay: clinical experiences for dabigatran monitoring. Thromb Haemost 2017;117(12): 2369-2375

15 Best Practice. Decision Support for Health Professionals. Available at: http://www.bestpractice.net.nz/. Accessed December 3, 2018

16 New Zealand Ministry of Health. Pharmaceutical Collection. 2016. Available at: https://www.health.govt.nz/nz-health-statistics/national-collections-and-surveys/collections/pharmaceutical-collection. Accessed June 27, 2019

17 Liesenfeld KH, Lehr T, Dansirikul C, et al. Population pharmacokinetic analysis of the oral thrombin inhibitor dabigatran etexilate in patients with non-valvular atrial fibrillation from the RE-LY trial. J Thromb Haemost 2011;9(11):2168-2175

18 U.S. Department of Health and Human Services Food and Drug Administration. Guidance for Industry. E7 Studies in Support of Special Populations: Geriatrics. Questions and Answers. 2012

19 Committee for Human Medicinal Products (CHMP). Adequacy of Guidance on the Elderly Regarding Medicinal Products for Human Use. European Medicines Agency. 2007

20 New Zealand Ministry of Health. National Minimum Dataset (Hospital Events) data dictionary. 2015

21 National Centre for Classification in Health (Australia). The International Statistical Classification of Diseases and Related Health Problems, 10th Revision, Australian modification (ICD-10-AM). 6th ed. Lidcombe, NSW: National Centre for Classification in Health; 2008

22 Coleman JS. Introduction to Mathematical Sociology. New York, NY: Free Press; 1964

23 Simpson BH, Reith D, Medlicott NJ, Smith A. Deprivation and inequalities lead to worse outcomes with dabigatran etexilate. J Prim Health Care 2018;10(04):303-311

24 Connolly SJ, Ezekowitz MD, Yusuf S, et al; RE-LY Steering Committee and Investigators. Dabigatran versus warfarin in patients with atrial fibrillation. N Engl J Med 2009;361(12):1139-1151

25 Reilly PA, Lehr T, Haertter S, et al; RE-LY Investigators. The effect of dabigatran plasma concentrations and patient characteristics on the frequency of ischemic stroke and major bleeding in atrial fibrillation patients: the RE-LY Trial (Randomized Evaluation of Long-Term Anticoagulation Therapy). J Am Coll Cardiol 2014;63 (04):321-328

26 Arnason T, Wells PS, van Walraven C, Forster AJ. Accuracy of coding for possible warfarin complications in hospital discharge abstracts. Thromb Res 2006;118(02):253-262

27 Woodfield R, Grant I, Sudlow CL; UK Biobank Stroke Outcomes Group; UK Biobank Follow-Up and Outcomes Working Group. Accuracy of electronic health record data for identifying stroke cases in large-scale epidemiological studies: A systematic review from the UK biobank stroke outcomes group. PLoS One 2015;10 (10): $\mathrm{e} 0140533$ 